\title{
PARTE 3: CASCAS
}

\section{GENERALIDADES}

Cascas são peças estruturais em que uma dimensão, a espessura $e$, é muito menor que as outras duas, não são necessariamente planas, e os carregamentos podem ser tanto tangenciais como normais à superfície da casca. É, genericamente, o caso de objetos como os painéis de fechamento de fuselagens de aeronaves e espaçonaves, veículos terrestres e navais, tubulações e vasos de pressão, silos, cúpulas etc.

\section{CASCAS DE REVOLUÇ̃̃OO COM CARGA AXISSIMÉTRICA}

Consideram-se, neste texto, apenas as cascas geradas por revolução de uma curva em torno de um eixo de rotação, conforme Fig. 3.1. Nesses casos, os raios de curvatura, normais à superfície em cada ponto da casca, e os respectivos centros de curvatura, situam-se no plano que contém esse ponto e o eixo de rotação. As curvas geradoras da superfície são denominadas meridianos, e os círculos descritos por um ponto A da curva rodando em torno do eixo são denominados paralelos, tal como no caso do planeta Terra. As longitudes são dadas por um 
ângulo $\theta$, medido a partir de uma linha de referência, arbitrariamente escolhida (como o meridiano de Greenwich na Terra), até o meridiano considerado, conforme Fig. 3.2, uma seção da casca normal ao eixo de rotação. Já a latitude, a posição do paralelo em questão, é dada por um ângulo $\phi$, conforme Fig. 3.3, uma seção da casca contendo um meridiano e o eixo de rotação, na qual também é definido o raio de curvatura do meridiano naquele ponto, $r_{1}$. Note-se nessa Figura que o raio de curvatura do paralelo em um ponto, $r_{2}$, está relacionado com o raio do círculo normal ao eixo de revolução nesse ponto nesse paralelo, $r_{0}$, por $r_{0}=r_{2} \operatorname{sem} \phi$.

Pequenas variações desses ângulos, $d \theta$ e $d \phi$, definem um elemento de casca, curvo nas 2 direções no caso geral, de área $d S$, com vértices A A' A", conforme Fig. 3.4.

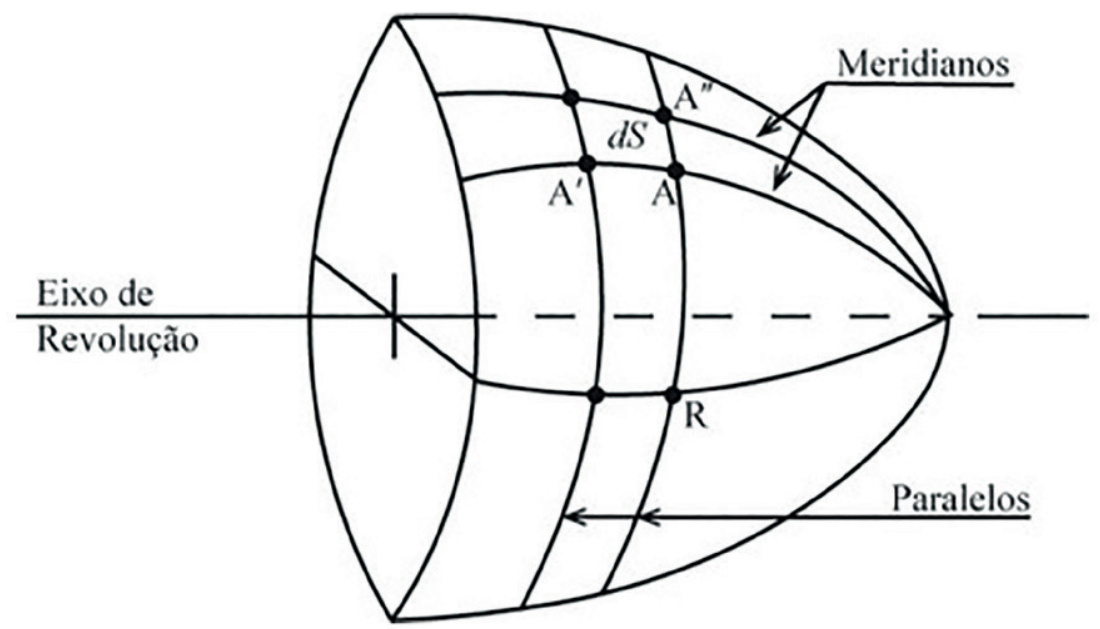

Figura 3.1: Uma casca de revolução

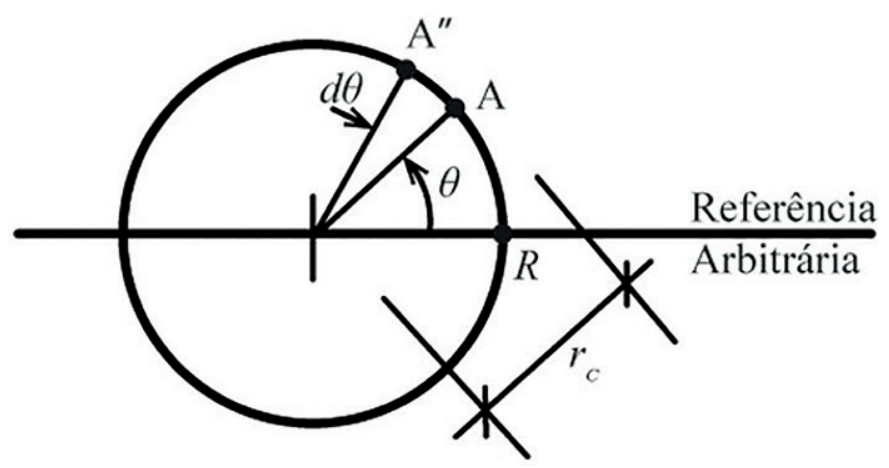

Figura 3.2: Posição dos meridianos 


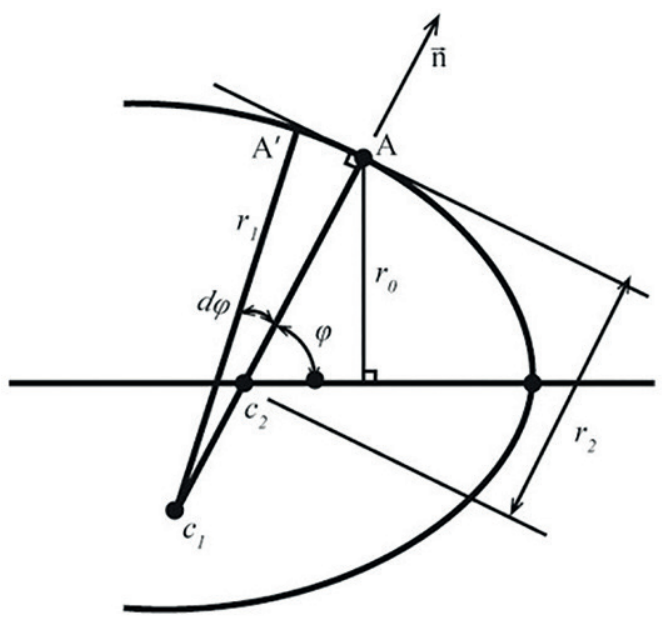

Figura 3.3: Posição dos paralelos

\subsection{Teoria de Membrana}

\subsubsection{Teoria}

Para determinados carregamentos (simétricos em relação ao eixo de rotação) e condições de contorno bastante comuns na prática, pode-se considerar que apenas forças normais atuam na casca, sem flexão. É a chamada Teoria de Membrana.

Nesta hipótese, no elemento de casca da Fig. 3.4 atuam apenas as forças $N_{\theta}$, na direção dos paralelos, e $N_{\phi}$, na direção dos meridianos. Essas forças têm unidades $\mathrm{N} / \mathrm{m}$. Além disso, tem-se o carregamento, que não necessariamente é normal ao elemento, que seria o caso de uma pressão, em N/m².

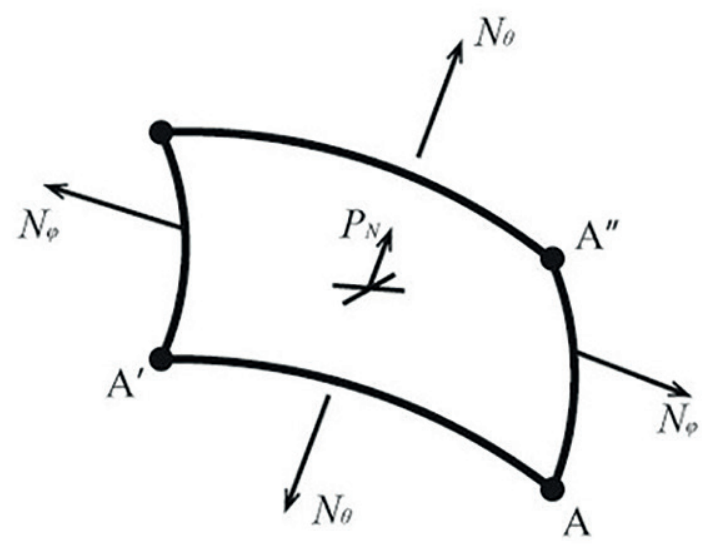

Figura 3.4: Elemento de casca 
O comprimento dos segmentos de curvas do perímetro do elemento de casca da Fig. 3.4 é ilustrado nas Figs. 3.2 e 3.3, e dados por

$$
\begin{gathered}
A^{\prime}=r_{1} d \phi \\
A A^{\prime \prime}=r_{0} d \theta
\end{gathered}
$$

Com esses comprimentos é possível calcular a área do elemento $r_{0} r_{1} d \theta d \phi$, e as resultantes das forças, na direção dos paralelos

$$
N_{\theta} r_{1} d \phi
$$

e na direção dos meridianos,

$$
N_{\phi} r_{0} d \theta=N_{\phi} r_{2} \operatorname{sen} \phi d \theta(N)
$$

A seguir, determina-se o equilíbrio de forças na direção normal ao elemento, em que atua a resultante normal do carregamento $p_{N} r_{0} r_{1} d \theta d \phi(\mathrm{N})$, considerando-se que para ângulos pequenos o seno é aproximadamente igual ao próprio ângulo em radianos,
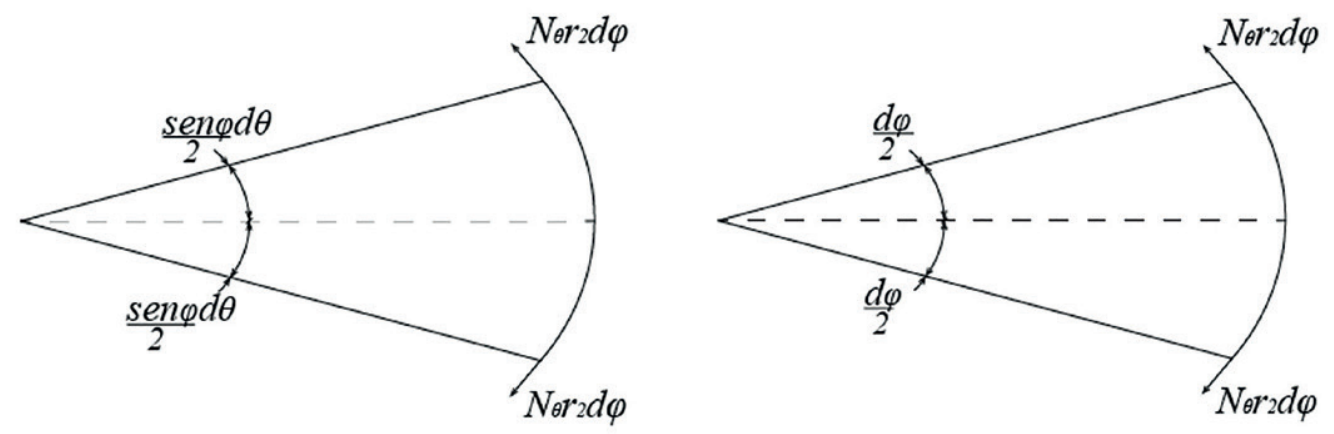

Figura 3.5: Decomposição de forças na direção normal à casca

$$
\begin{aligned}
& 2 N_{\phi} r_{0} d \theta \operatorname{sen} \frac{d \phi}{2}+2 N_{\theta} r_{1} d \phi \operatorname{sen} \frac{\operatorname{sen} \phi d \theta}{2}-p_{N} r_{0} r_{1} d \theta d \phi \\
& =0 N_{\phi} r_{0} d \theta d \phi+N_{\theta} r_{1} d \theta d \phi \operatorname{sen} \phi-p_{N} r_{0} r_{1} d \theta d \phi=0
\end{aligned}
$$


e, dividindo-se por $r_{0} r_{1} d \theta d \phi$, chega-se a

$$
\frac{N_{\phi}}{r_{1}}+\frac{N_{\theta}}{r_{2}}=p_{N}
$$

Nesta equação, têm-se 2 incógnitas. A outra equação necessária, pode ser obtida de várias maneiras. A mais simples é considerar um corte na casca contendo o paralelo em análise, e determinar o equilíbrio entre a resultante $R$ do carregamento sobre a parte da casca de um lado do paralelo, na direção do eixo de rotação, e as forças $N_{\phi}$ ao longo da circunferência de comprimento $2 \pi r_{0}$, as quais têm a inclinação $\phi$ com relação ao eixo de rotação, conforme Fig. 3.5. Obtêm-se

$$
\begin{gathered}
2 \pi r_{0} N_{\phi} \cos \left(\frac{\pi}{2}-\phi\right)-R=0 \text { ou } \\
N_{\phi}=\frac{R}{2 \pi r_{0} \operatorname{sen} \phi}=\frac{\pi r_{0}^{2} p}{2 \pi r_{0} \operatorname{sen} \phi}=\frac{p r_{0}}{2 \operatorname{sen} \phi}=\frac{p r_{2}}{2}
\end{gathered}
$$

Tendo-se as forças por unidade de comprimento, basta dividi-las pela espessura da casca $e$ para serem obtidas as tensões normais atuantes nas duas direções.

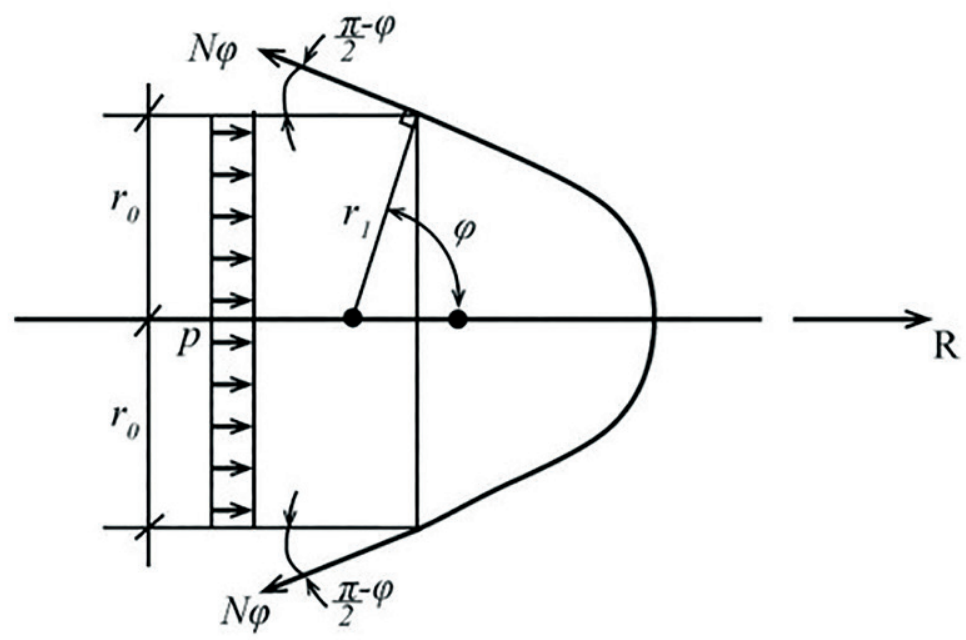

Figura 3.6: Obtenção da força na direção dos meridianos 


\section{EXEMPLOS}

\subsection{Balão esférico}

Considera-se um balão esférico sob pressão interna de gás $p$, de raio $r$ e espessura $e$.

Os raios de curvatura são iguais

$$
r_{1}=r_{2}=r
$$

e, da Eq. 3.6, e levando em conta que $N_{\phi}=N_{\theta}$, por simetria,

$$
N_{\phi}=N_{\theta}=\frac{p r}{2}
$$

As tensões normais principais atuantes valem

$$
\sigma_{\phi}=\sigma_{\theta}=\frac{p r}{2 e}
$$

\subsection{Casca cilíndrica (fuselagem de aeronave ou tubulação, pressurizada)}

Considera-se uma casca cilíndrica, fechada nas extremidades, de raio $r$ e espessura $e$ sob pressão interna $p$.

Neste caso, como a curva geradora da casca é uma reta paralela ao eixo de revolução, o raio de curvatura do meridiano $r_{1}$ é infinito (curvatura nula) e o raio de curvatura do paralelo $r_{2}=r$.

A resultante de pressão sobre as extremidades, conforme Eq. 3.7, é

$$
\mathrm{R}=\mathrm{p} \pi \mathrm{r}
$$

que dividida pelo perímetro $2 \pi r$ resulta a força por unidade de comprimento

$$
\mathrm{N}_{\phi}=\frac{\mathrm{pr}}{2}(\mathrm{~N} / \mathrm{m})
$$


paralela ao eixo do cilindro. Da Eq. 3.6, obtém-se

$$
N_{\theta}=p r
$$

As tensões normais principais atuantes valem

$$
\sigma_{\theta}=2 \sigma_{\phi}=\frac{p r}{e}
$$

\subsection{Casca cônica}

Considera-se uma casca em forma de cone de raio na base $a$, altura $b$ e espessura $e$, sob pressão interna de gás $p$, gerado pela revolução de uma reta em torno do eixo $y$. A tangente da geratriz em relação ao eixo $y$ é $\phi=\operatorname{arctg}\left(\frac{b}{a}\right)$. Os resultados são:

$$
r_{1}=\infty \quad r_{2}=-\frac{x}{\operatorname{sen} \phi} \quad N_{\phi}=\frac{p r_{2}}{2} \quad N_{\theta}=p r_{2}
$$

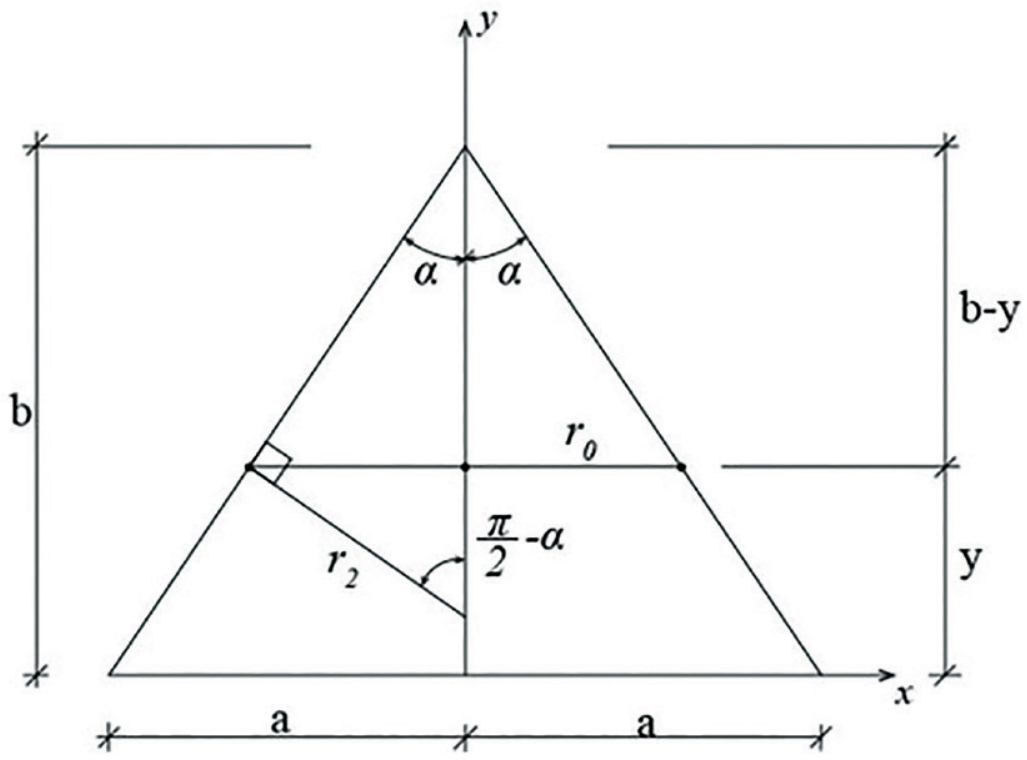

Figura 3.7: Casca cônica 


\subsection{Paraboloide}

Considera-se um paraboloide de revolução em torno do eixo $y$, de raio na base $a$, altura $b$ e espessura $e$, sob pressão interna de gás $p$.

$$
\begin{gathered}
y=b\left(1-\frac{x^{2}}{a^{2}}\right) \quad y^{\prime}=-\frac{2 b x}{a^{2}} \quad y^{\prime \prime}=-\frac{2 b}{a^{2}} \\
r_{1}=-\frac{\sqrt{\left[1+\left(y^{\prime}\right)^{2}\right]^{3}}}{y^{\prime \prime}} \quad r_{2}=\frac{|x|}{\operatorname{sen} \phi}
\end{gathered}
$$

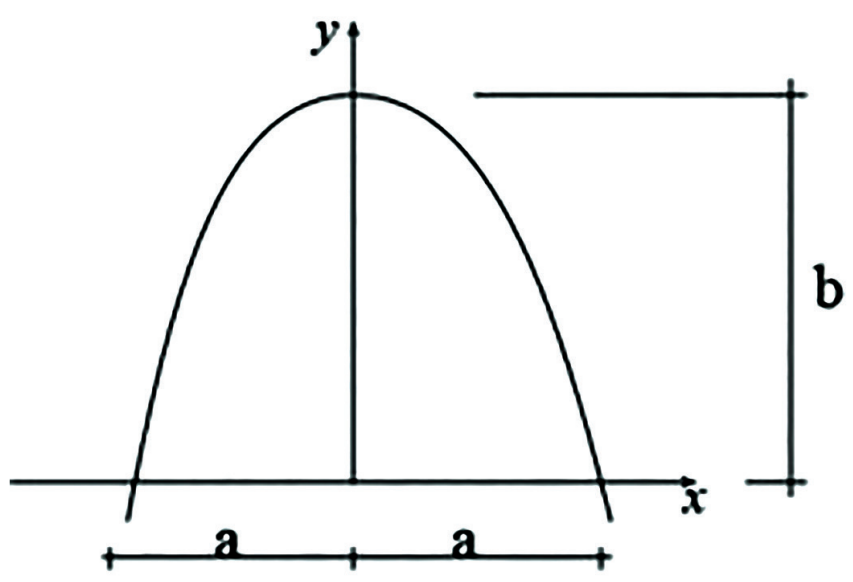

Figura 3.8: Paraboloide de revolução

\subsection{Dirigível elíptico ou extremidade de vaso de pressão cilíndrico}

Considera-se uma casca em forma de um elipsoide de revolução de raio maior $a$, raio menor $b$ e espessura $e$, sob pressão interna de gás $p$.

Uma aplicação seria um dirigível sob pressão do gás interno. Outra, bastante comum, é usar a metade de um elipsoide de revolução como extremidades de um vaso de pressão cilíndrico, como as extremidades de uma fuselagem de aeronave.

Os raios de curvatura principais dessa casca, em função do ângulo $\phi$, indicado na Figura 3.6, conforme a Geometria Diferencial, valem 


$$
\begin{gathered}
y=b \sqrt{1-\frac{x^{2}}{a^{2}}} \quad y^{\prime}=-\frac{b x}{a^{2} \sqrt{1-\frac{x^{2}}{a^{2}}}} \quad y^{\prime \prime}=-\frac{b}{a^{2} \sqrt{\left(1-\frac{x^{2}}{a^{2}}\right)^{3}}} \\
\boldsymbol{r}_{1}=-\frac{\sqrt{\left[1+\left(y^{\prime}\right)^{2}\right]^{3}}}{y^{\prime \prime}} \quad r_{2}=\frac{|x|}{\operatorname{sen} \phi}
\end{gathered}
$$

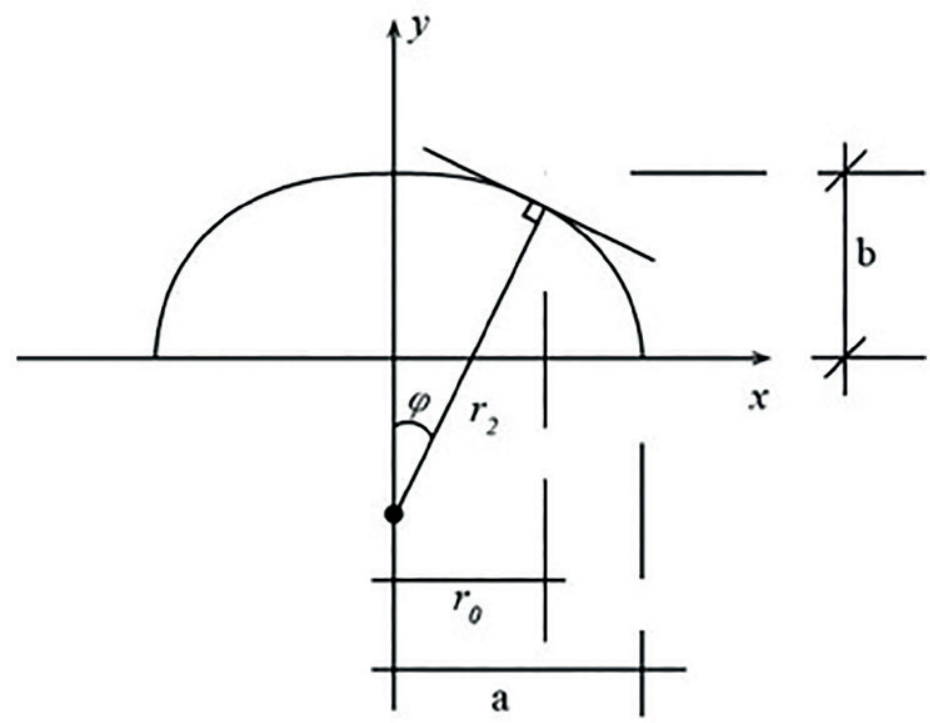

Figura 3.9: Raios de curvatura de elipsoide de revolução

Dados os raios de curvatura, é possível obter as forças $N_{\phi}$ e $N_{\theta}$ pelas Equações 3.6 e 3.7 .

Uma análise mais expedita é feita para um paralelo de raio $r_{0}$. A resultante mencionada na Eq. 3.7 vale $R=\pi p r_{0}^{2}$, levando a

$$
N_{\phi}=\frac{p r_{0}}{2 \operatorname{sen} \phi}=\frac{p r_{2}}{2}
$$

Substituindo esse valor na Eq. 3.6, encontra-se

$$
N_{\theta}=r_{2} p-\frac{r_{2}}{r_{1}} N_{\phi}=p\left(r_{2}-\frac{r_{2}^{2}}{2 r_{1}}\right)
$$


No topo da casca, $r_{1}=r_{2}=\frac{a^{2}}{b}$, de forma que

$$
N_{\phi}=N_{\theta}=\frac{p a^{2}}{2 b}
$$

No equador da casca, $r_{1}=\frac{b^{2}}{a}$ e $r_{2}=a$, de forma que

$$
N_{\phi}=\frac{p a}{2} \text { e } N_{\theta}=p a\left(1-\frac{a^{2}}{2 b^{2}}\right)
$$

Assim, $N_{\phi}$ é sempre positiva, enquanto $N_{\theta}$ fica negativa no equador se $a^{2}>2 b^{2}$.

\section{TEORIA FLEXIONAL PARA CASCAS CILÍNDRICAS}

Em muitas aplicações práticas têm-se cascas cilíndricas carregadas simetricamente em relação ao eixo. É, e.g., o caso de vasos de pressão cilíndricos (como fuselagens de aeronaves), tubulações (como raiser de petróleo em instalações off-shore) etc.

Considere-se o elemento de casca cilíndrica de espessura $e$ da Fig. 3.10, em que o eixo longitudinal é $x$, o eixo $y$ é tangente à casca em um ponto e o eixo $z$, normal a esses eixos, tem a direção do raio do cilindro. Como a curva geradora da casca é uma reta paralela ao eixo, o raio de curvatura do meridiano $r_{1}$ é infinito (curvatura nula) e o raio de curvatura do paralelo $r_{2}=a$.

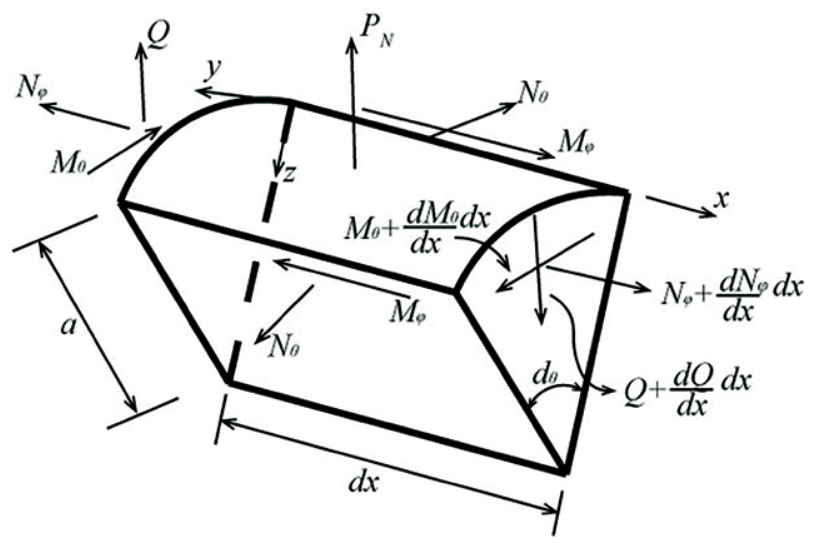

Figura 3.10: Elemento de casca cilíndrica 
Por razões de simetria, não há forças tangenciais $N_{\theta \phi}=N_{\phi \theta}$, só havendo forças tangenciais $Q$. Ainda por simetria, não há momentos de torção $M_{x \phi}=M_{\phi x}$, e o momento fletor $M_{\phi}$ tem que ser constante ao longo da circunferência. Das 6 equações de equilíbrio em $3 \mathrm{D}$, restam apenas 3 :

$$
\begin{aligned}
& \sum F_{x}=0=\frac{d N_{\phi}}{d x} a d x d \theta \\
& \sum F_{z}=0=\frac{d Q_{x}}{d x} a d x d \theta+N_{\theta} d x d \theta+p_{N} d x d \theta \\
& \sum M_{y}=0=\frac{d M_{\theta}}{d x} a d x d \theta-Q_{x} a d x d \theta
\end{aligned}
$$

A primeira equação de (3.8) implica em que $N_{\phi}$ é constante, e facilmente determinável na prática, não afetando o cálculo dos momentos, podendo ser considerada nula nas derivações que se seguem. Restam

$$
\begin{aligned}
& \frac{d Q_{x}}{d x}+\frac{1}{a} N_{\phi}=-p_{N} \\
& \frac{d M_{x}}{d x}-Q_{x}=0
\end{aligned}
$$

duas equações e três incógnitas. Para solução, considera-se os deslocamentos no sentido longitudinal $u$ e o no sentido radial $w$, segundo Fig. 3.11.

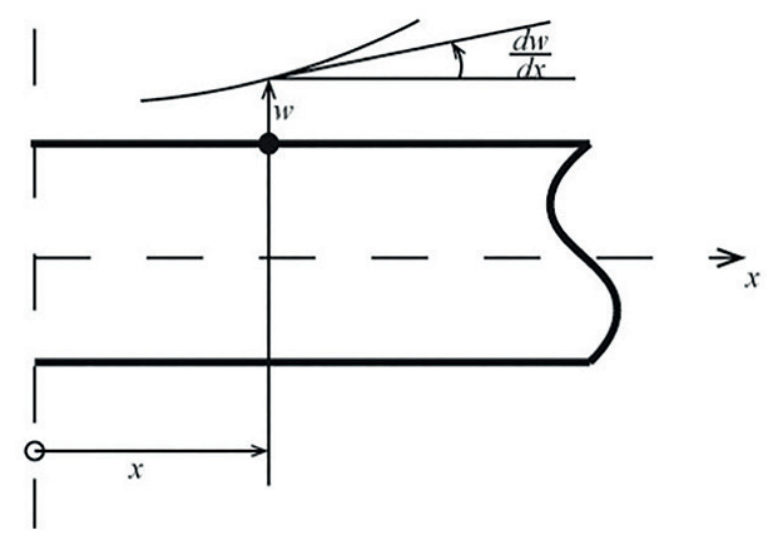

Figura 3.11: Deslocamento radial da casca 
As deformações nesses dois sentidos são

$$
\varepsilon_{x}=\frac{d u}{d x} \text { e } \varepsilon_{\phi}=-\frac{w}{a}
$$

levando, por meio da Lei de Hooke, a

$$
\frac{d u}{d x}=v \frac{w}{a}
$$

Da segunda equação de equilíbrio

$$
N_{\phi}=-\frac{E t w}{a}
$$

Por simetria, não há variação na curvatura na direção circunferencial. Já a curvatura na direção longitudinal é $-\frac{d^{2} w}{d x^{2}}$. Lembrando as considerações feitas na dedução das expressões dos momentos fletores em placas planas, chega-se a

$$
\begin{gathered}
M_{\phi}=v M_{x} \\
M_{\theta}=-D \frac{d^{2} w}{d x^{2}} \quad \text { onde } \quad D=\frac{E t^{3}}{12\left(1-v^{2}\right)}
\end{gathered}
$$

Retornando às equações de equilíbrio (3.9), e supondo a espessura $t$ constante, obtém-se

$$
\frac{d^{4} w}{d x^{4}}+4 \beta^{4} w=\frac{p_{N}}{D} \quad \text { onde } \quad \beta=\frac{E t}{4 a^{2} D}=\frac{3\left(1-v^{2}\right)}{a^{2} t^{2}}
$$

A Eq. (3.15) é a equação diferencial que rege o problema. A solução geral padrão para esse tipo de equação diferencial é a soma da solução homogênea com a particular:

$$
w=e^{\beta x}\left(C_{1} \cos \beta x+C_{2} \operatorname{sen} \beta x\right)+e^{-\beta x}\left(C_{3} \cos \beta x+C_{4} \operatorname{sen} \beta x\right)+f(x)
$$

$\mathrm{Na}$ Eq. (3.16) as constantes saem das condições de contorno geométricas nas extremidades do cilindro. 


\subsection{Exemplos}

\subsubsection{Casca cilíndrica}

Verificar que a solução do exemplo 3.2. atende a Eq. 3.15.

\subsubsection{Momento e força cortante distribuídos na borda de uma casca cilíndrica}

Considera-se a Fig. 3.12, representando um carregamento composto de momento fletor por unidade de comprimento $M_{0}(\mathrm{Nm} / \mathrm{m})$ e força cortante por unidade de comprimento $Q_{0}(\mathrm{~N} / \mathrm{m})$ uniformemente distribuídos no perímetro da extremidade de uma casca cilíndrica longa, não pressurizada interiormente $\left(p_{\mathrm{N}}=0\right)$, implicando que não há solução particular $f(x)$ na Eq. (3.16).

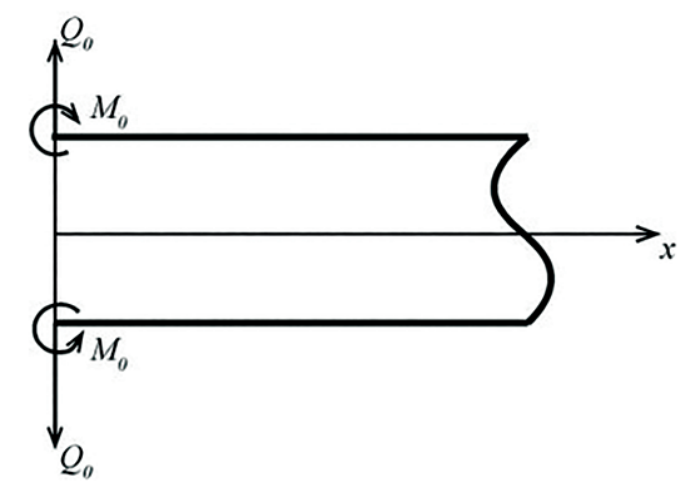

Figura 3.12: Casca cilíndrica com momentos e forças por unidade na borda

Como fisicamente em cascas finas o momento fletor deve diminuir rapidamente com a variável $x$, é necessário que $C_{1}=C_{2}=0$, restando

$$
w=e^{-\beta x}\left(C_{3} \cos \beta x+C_{4} \operatorname{sen} \beta x\right)
$$

No contorno

$$
\begin{gathered}
\left(M_{\theta}\right)_{x=0}=-D\left(\frac{d^{2} w}{d x^{2}}\right)_{x=0}=M_{0} \\
(\mathrm{Q})_{\mathrm{x}=0}=\left(\frac{\mathrm{dM}_{\mathrm{x}}}{\mathrm{dx}}\right)_{\mathrm{x}=0}=-\mathrm{D}\left(\frac{\mathrm{d}^{3} \mathrm{w}}{\mathrm{dx}^{3}}\right)_{\mathrm{x}=0}=\mathrm{Q}_{0}
\end{gathered}
$$


resultando

$$
C_{3}=-\frac{1}{2 \beta^{3} D}\left(Q_{0}+M_{0}\right) \quad C_{4}=\frac{M_{0}}{2 \beta^{2} D}
$$

$\mathrm{e}$

$$
w=\frac{e^{-\beta x}}{2 \beta^{3} D}\left[\beta M_{0}(\operatorname{sen} \beta x-\cos \beta x)-Q_{0} \cos \beta x\right]
$$

É simples verificar, plotando em uma planilha EXCEL, por exemplo, ou no MATLAB, que o valor máximo é obtido na borda carregada $x=0$ e vai diminuindo rapidamente ao longo desse eixo.

\section{ElEMENTOS FINITOS PARA CASCAS de REVOLUÇ̃̃O}

Muitos problemas tridimensionais no campo da engenharia apresentam simetria ao redor de um eixo de rotação, tanto em sua geometria como nas condições de contorno e cargas aplicadas.

Esses problemas são conhecidos como axissimétricos. Eles podem ser analisados de maneira mais simples como um caso bidimensional no plano $r z$, em que $r$ é o raio $\mathrm{e} z$ a coordenada longitudinal. Os resultados são equivalentes à resposta global do sólido de revolução. A Figura 3.13 mostra um sólido de revolução sujeito a cargas distribuídas simétricas com respeito ao eixo vertical $\mathrm{Z}$ indicando a simplificação realizada para análise pelo MEF como um caso bidimensional.
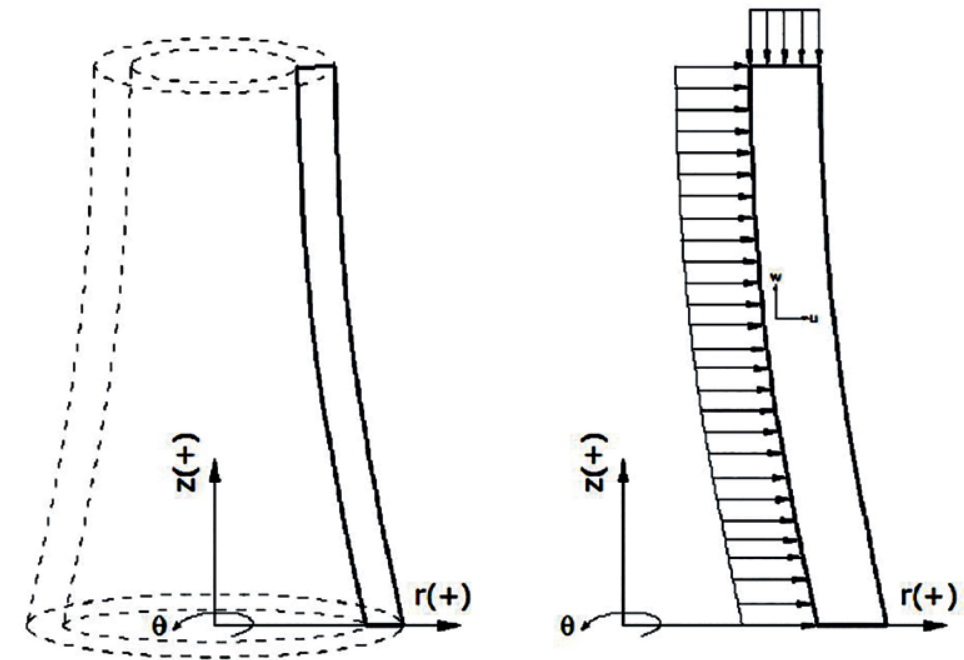

Figura 3.13: Estrutura genérica com simetria axial

Fonte: Guallichico S. F. R., \& Macas V. X. O. (2010). 
Vantagens:

- malhas de elementos finitos mais simples.

- redução significativa do número de graus de liberdade.

- diminuição substancial do tempo de resolução do sistema de equações.

- maior facilidade de interpretação de resultados.

\subsection{Mecânica de sólidos axissimétricos}

\subsubsection{Deslocamentos e deformações}

Em um sólido que cumpre com as condições para ser modelado como um sólido de revolução, o movimento de um ponto qualquer localizado na secção caraterística de revolução pode ser perfeitamente definido pelas componentes de deslocamento radial $u$ e axial $w$, como mostra a Figura 3.14.

$$
\{u\}=\left\{\begin{array}{l}
u(r, z) \\
w(r, z)
\end{array}\right\}
$$

O vetor das deformações de um ponto, neste caso, tem as seguintes componentes:

1. $\varepsilon_{r}$ : deformação radial.

2. $\varepsilon_{z}$ : deformação axial.

3. $\varepsilon_{\theta}$ : deformação circunferencial.

4. $\gamma_{r z}$ : deformação tangencial.

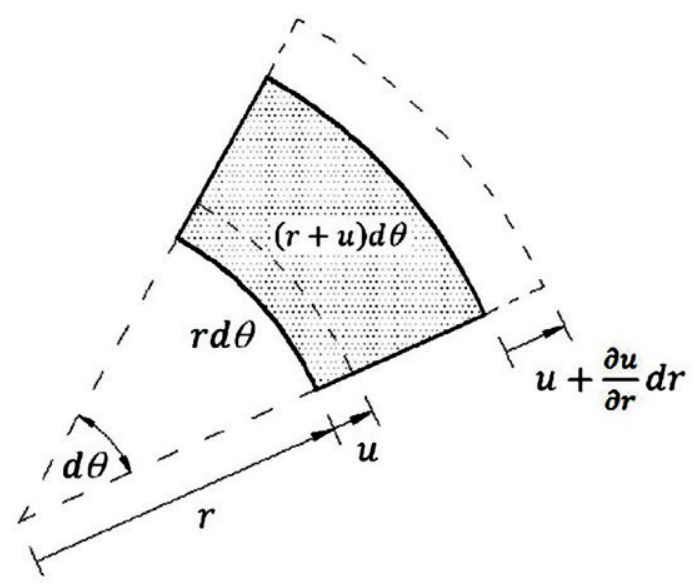

Figura 3.14: Elemento diferencial circunferencial 
Analisando a Figura 3.14 pode-se ver que a deformação radial tem a forma:

$$
\varepsilon_{r}=\frac{1}{d r}\left(u+\frac{\partial u}{\partial r} d r-u\right)=\frac{\partial u}{\partial r}
$$

De maneira semelhante, a deformação axial é dada pela relação

$$
\varepsilon_{z}=\frac{1}{d z}\left(w+\frac{\partial w}{\partial z} d z-w\right)=\frac{\partial w}{\partial z}
$$

Por outro lado, a deformação circunferencial está estreitamente relacionada com a deformação radial, expressando-se da forma:

$$
\varepsilon_{\theta}=\frac{(r+u) d \theta-r d \theta}{r d \theta}=\frac{r}{u}
$$

De acordo com estas 3 equações, pode-se escrever o vetor de deformações:

$$
\{\varepsilon\}=\left\{\begin{array}{c}
\varepsilon_{r} \\
\varepsilon_{\theta} \\
\varepsilon_{z} \\
\gamma_{r z}
\end{array}\right\}=\left\{\begin{array}{c}
\frac{\partial u}{\partial r} \\
\frac{u}{r} \\
\frac{\partial w}{\partial z} \\
\frac{\partial u}{\partial z}+\frac{\partial w}{\partial r}
\end{array}\right\}
$$

ou

$$
\{\varepsilon\}=[L]\{u\}
$$

Para este caso, a matriz de operadores diferenciais $[L]$ teria a forma:

$$
[L]=\left[\begin{array}{cc}
\frac{\partial}{\partial r} & 0 \\
\frac{1}{r} & 0 \\
0 & \frac{\partial}{\partial z} \\
\frac{\partial}{\partial z} & \frac{\partial}{\partial r}
\end{array}\right]
$$




\subsubsection{Tensões}

As tensões não nulas, correspondentes às deformações não nulas, são mostradas em um elemento diferencial sujeito ao estado de tensões mostrado na Figura 3.15.

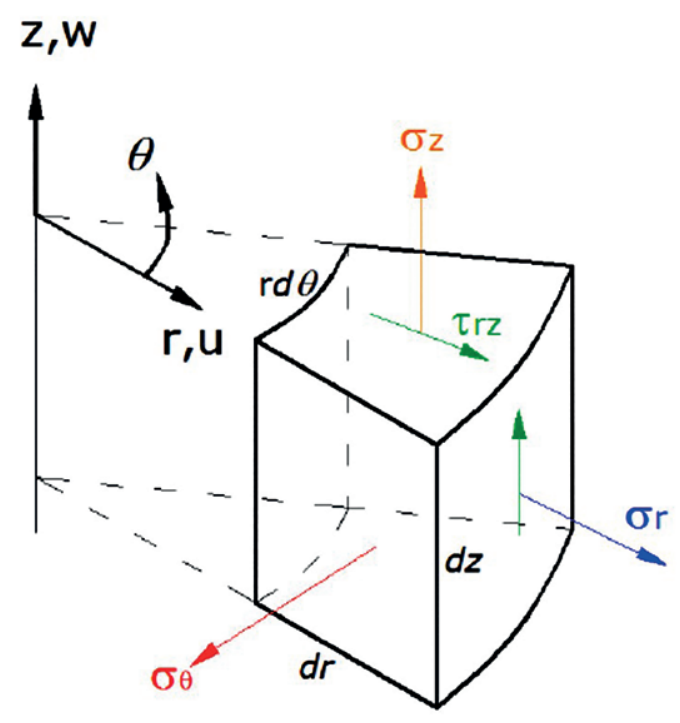

Figura 3.15: Tensões atuando sobre um elemento diferencial de um sólido de revolução

Então o vetor do campo de tensões tem a forma:

$$
\{\sigma\}=\left\{\begin{array}{c}
\sigma_{r} \\
\sigma_{\theta} \\
\sigma_{z} \\
\tau_{r z}
\end{array}\right\}
$$

onde $\sigma_{r}, \sigma_{z}, \sigma_{\theta}, \tau_{r z}$ são, respectivamente, as tensões radial, axial, circunferencial e tangencial.

\subsubsection{Equação constitutiva}

Considerando um material isotrópico e homogêneo, cujo comportamento se encontra na região linear (lei de Hooke), a equação constitutiva tem a forma:

$$
\{\sigma\}=[D]\{\varepsilon\}
$$


Em consequência, tem-se:

$$
\left\{\begin{array}{c}
\sigma_{r} \\
\sigma_{\theta} \\
\sigma_{z} \\
\tau_{r z}
\end{array}\right\}=\frac{E}{(1+v)(1-2 v)}\left[\begin{array}{cccc}
1-v & \vartheta & \vartheta & 0 \\
\vartheta & 1-v & \vartheta & 0 \\
\vartheta & \vartheta & 1-v & 0 \\
0 & 0 & 0 & \frac{1-2 v}{2}
\end{array}\right]\left\{\begin{array}{c}
\varepsilon_{r} \\
\varepsilon_{\theta} \\
\varepsilon_{z} \\
\gamma_{r z}
\end{array}\right\}
$$

onde $[D]$, a matriz de propriedades do material para o caso bidimensional é:

$$
[D]=\frac{E}{(1+v)(1-2 v)}\left[\begin{array}{cccc}
1-v & v & v & 0 \\
\vartheta & 1-v & v & 0 \\
\vartheta & \vartheta & 1-v & 0 \\
0 & 0 & 0 & \frac{1-2 v}{2}
\end{array}\right]
$$

\subsection{Discretização por elementos finitos}

Supõem-se, agora, que o domínio do sólido foi discretizado em elementos pequenos, porém finitos. $\mathrm{O}$ vetor de deslocamentos nodais para um elemento bidimensional de $M$ nós é dado pela equação

$$
\left[q^{(e)}\right]=\left\{\begin{array}{c}
{\left[\begin{array}{l}
u_{1} \\
w_{1}
\end{array}\right]} \\
{\left[\begin{array}{l}
u_{2} \\
w_{2}
\end{array}\right]} \\
\cdots \\
{\left[\begin{array}{l}
u_{M} \\
w_{M}
\end{array}\right]}
\end{array}\right\}
$$

Adota-se uma matriz de funções de forma para este elemento, ordenadas na forma:

$$
N=\left(\left[\begin{array}{cc}
N_{1} & 0 \\
0 & N_{1}
\end{array}\right] \quad\left[\begin{array}{cc}
N_{2} & 0 \\
0 & N_{2}
\end{array}\right] \quad \cdots \quad\left[\begin{array}{cc}
N_{M} & 0 \\
0 & N_{M}
\end{array}\right]\right)
$$

permitindo chegar a um campo de deslocamentos aproximado da forma:

$$
\begin{aligned}
& u(r, z)=\sum_{i=1}^{M} N_{i}(r, z) u_{i} \\
& w(r, z)=\sum_{i=1}^{M} N_{i}(r, z) w_{i}
\end{aligned}
$$


ou, em forma matricial:

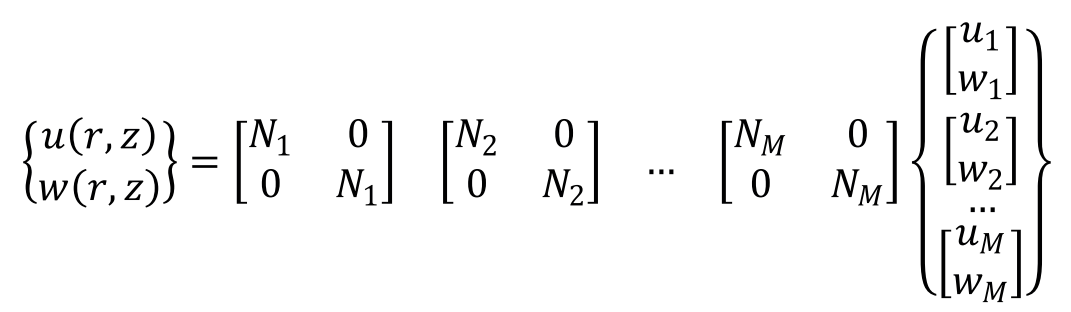

Do produto da matriz de operadores diferenciais pela matriz de funções de forma, resulta a matriz gradiente de deformação $[B]$

$$
[B]=[N][L]=\left[\begin{array}{cc}
\frac{\partial}{\partial r} & 0 \\
\frac{1}{r} & 0 \\
0 & \frac{\partial}{\partial z} \\
\frac{\partial}{\partial z} & \frac{\partial}{\partial r}
\end{array}\right]\left[\begin{array}{cc}
N_{1} & 0 \\
0 & N_{1}
\end{array}\right]\left[\begin{array}{cc}
N_{2} & 0 \\
0 & N_{2}
\end{array}\right] \cdots\left[\begin{array}{cc}
N_{M} & 0 \\
0 & N_{M}
\end{array}\right]
$$

que também pode ser expressa na forma:

$$
[B]=\left\{\left[\begin{array}{cc}
\frac{\partial N_{1}}{\partial r} & 0 \\
\frac{N_{1}}{r} & 0 \\
0 & \frac{\partial N_{1}}{\partial z} \\
\frac{\partial N_{1}}{\partial z} & \frac{\partial N_{1}}{\partial r}
\end{array}\right]\left[\begin{array}{cc}
\frac{\partial N_{2}}{\partial r} & 0 \\
\frac{N_{2}}{r} & 0 \\
0 & \frac{\partial N_{2}}{\partial z} \\
\frac{\partial N_{2}}{\partial z} & \frac{\partial N_{2}}{\partial r}
\end{array}\right] \cdots\left[\begin{array}{cc}
\frac{\partial N_{M}}{\partial r} & 0 \\
\frac{N_{M}}{r} & 0 \\
0 & \frac{\partial N_{M}}{\partial z} \\
\frac{\partial N_{M}}{\partial z} & \frac{\partial N_{M}}{\partial r}
\end{array}\right]\right\}
$$

permitindo a aproximação para as deformações:

$$
\varepsilon=L U=L N U^{(e)}=B U^{(e)}
$$




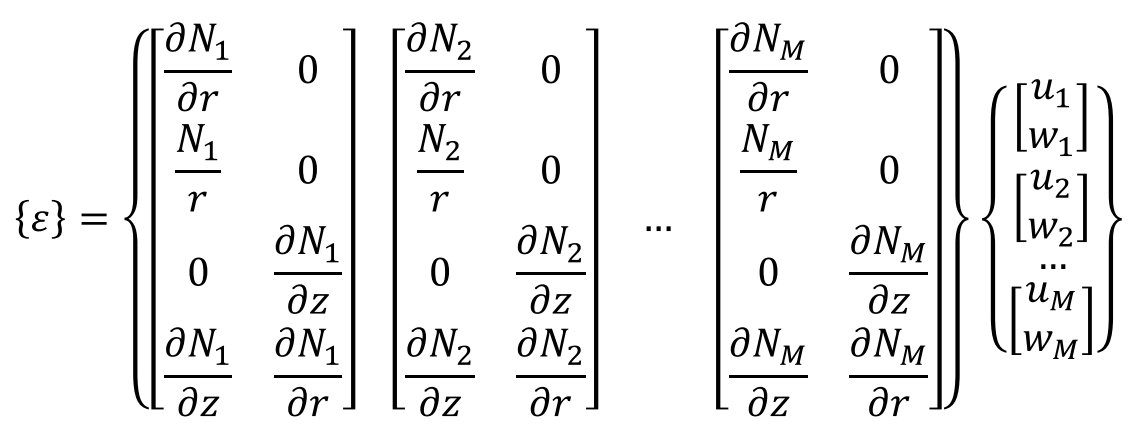

Da aproximação do campo de deformações resulta a aproximação do campo de tensões:

$$
\{\sigma\}=[D][B]\left[U^{(e)}\right]
$$

A seguir, pela aplicação de um princípio físico, como o do mínimo da Energia Potencial Total ou dos Trabalhos Virtuais, ou uma técnica matemática de minimização de resíduos, como a de Galerkin, chega-se à matriz de rigidez do elemento.

Essa matriz contém a resposta interna do sistema que equilibra as solicitações externas. Sendo assim, a matriz de um elemento genérico é dada pela expressão:

$$
\begin{aligned}
& {\left[k^{(e)}\right]=\int_{V^{(e)}}[B]^{T}[D][B] d V^{(e)} M_{\theta}=-D \frac{d^{2} w}{d x^{2}}} \\
& \text { onde } D=\frac{E t^{3}}{12\left(1-v^{2}\right)}
\end{aligned}
$$

com

$$
\begin{aligned}
& d V^{(e)}=2 \pi r d r d z M_{\theta}=-D \frac{d^{2} w}{d x^{2}} \\
& \text { onde } D=\frac{E t^{3}}{12\left(1-v^{2}\right)}
\end{aligned}
$$


Assim, a matriz de rigidez do elemento terá a forma:

$$
\begin{aligned}
& {\left[k^{(e)}\right]=2 \pi \int_{A^{(e)}}[B]^{T}[D][B] r d r d z M_{\theta}=-D \frac{d^{2} w}{d x^{2}}} \\
& \text { onde } \quad D=\frac{E t^{3}}{12\left(1-v^{2}\right)}
\end{aligned}
$$

A integração explícita desta última expressão não é recomendada devido à sua complexidade. Geralmente, é utilizada a técnica de integração numérica de Gauss, a qual se efetua em pontos especificamente determinados para o elemento finito em questão. Uma outra forma de fazer este cálculo de forma aproximada é avaliar a matriz $[B]$ no centroide do elemento com o qual as matrizes da integral se transformam em constantes e a matriz de rigidez aproximada é expressa por:

$$
\left[k^{(e)}\right]=2 \pi r A[\bar{B}]^{T}[D][\bar{B}]
$$

onde $2 \pi r A$ é o volume do elemento axissimétrico.

A matriz do elemento é quadrada e simétrica, de dimensões $n \times n$, em que $n$ é o produto dos graus de liberdade por nó do elemento pelo número de nós $M$.

No caso dinâmico, as matrizes de massa dos elementos podem ser obtidas a partir do vetor de velocidades aproximadas obtidas pela derivação no tempo do vetor de deslocamentos aproximado.

No passo seguinte, é feito o chamado "espalhamento" em que os coeficientes das matrizes dos elementos são adicionados às matrizes de rigidez e massa globais da estrutura nas posições dadas pela conectividade previamente definida no modelo.

Ao final, a solução das equações de equilíbrio global fornece os deslocamentos nodais dos quais se podem determinar os deslocamentos, deformações e tensões no interior dos elementos. 


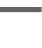




\section{REFERÊNCIAS BIBLIOGRÁFICAS}

Brasil, R.M.L.R.F, Bases da Mecânica dos Sólidos Elásticos, com elementos finitos. Editora da UFABC, Santo André, 2017.

Brasil, R.M.L.R.F., Silva, M.A., Introdução à Dinâmica das Estruturas para a Engenharia Civil. Editora Edgard Blucher, São Paulo, 2013.

Leissa, A.W., Vibration of Plates. NASA, Washington, 1969.

Ogden, J.T., Reddy, J.N., An Introduction to the Mathematical Theory of Finite Elements. Dover Publications, Mineola, 2011.

Reddy, J.N., Theory and Analysis of Elastic Plates and Shells, 2nd Ed. CRC Press, Boca Raton, 2007.

Timoshenko, S.P., Woinowsky-Krieger, S., Theory of Plates and Shells. McGraw-Hill Books, New York, 1964. 
Timoshenko, S.P., Goodier, J.N., Theory of Elasticity, 3rd Ed. McGraw-Hill Books, New York, 2010.

Timoshenko, S.P., Gere, J., Theory of Elastic Stability, 2nd Ed. McGraw-Hill Books, New York, 1989.

Zagottis, D.L., Introdução à Teoria das Placas e Cascas. Escola Politécnica da USP, São Paulo, 1973. 